\title{
Nucleotide Sequencing and Phylogenetic Analysis Using PCR Amplicons of $U 3$ Gene of Jaagsiekte Sheep Retrovirus (JSRV) Detected in Natural Cases of Ovine Pulmonary Adenocarcinoma in India
}

\author{
Vemuri Rama Devi', Egi Janardhan Yadav', Tumati Sreenivasa Rao², Kokila Satheesh', \\ Paila Suresh', Betala Baby Manasa ${ }^{1}$ \\ ${ }^{1}$ Department of Veterinary Pathology, N.T.R. College of Veterinary Science, Gannavaram, India \\ ${ }^{2}$ Department of Veterinary Public Health, N.T.R. College of Veterinary Science, Gannavaram, India \\ Email: vrdpath@yahoo.com
}

Received 26 September 2014; revised 28 October 2014; accepted 10 November 2014

Copyright (C) 2014 by authors and Scientific Research Publishing Inc.

This work is licensed under the Creative Commons Attribution International License (CC BY).

http://creativecommons.org/licenses/by/4.0/

cc) (i) Open Access

\begin{abstract}
Ovine pulmonary adenocarcinoma (OPA) caused by an exogenous Jaagsiekte sheep retrovirus (JSRV) is prevalent in Indian sheep. In the present study, OPA was diagnosed in sheep by clinical signs, gross and histopathology and polymerase chain reaction (PCR). Proviral DNA of exogenous JSRV was detected in lung tumor tissues, mediastinal lymph nodes, blood and lung fluid samples from natural cases of OPA by using U3-hn PCR and the PCR amplicons were sequenced to analyze nucleotide divergence. In total, six isolates were sequenced that had $96 \%-100 \%$ homology with a UK strain (AF105220.1) but more divergent from a South African strain (M80216) with 88\% - 93\% identity. The phylogenetic analysis revealed segregation of the six isolates into two clusters. In conclusion, this study is the first report on sequencing and phylogenetic analysis of JSRV in India and further studies are suggested to know the complete sequencing and genetic divergence of JSRV in Indian sheep.
\end{abstract}

\section{Keywords}

OPA, JSRV, Sequencing, Phylogenesis, India 


\section{Introduction}

Ovine pulmonary adenocarcinoma (OPA), a contagious lung cancer of sheep caused by an exogenous retrovirus known as Jaagsiekte sheep retrovirus (JSRV), is prevalent in many parts of India resulting in economic losses [1]-[4]. But it could not receive considerable importance in the control and eradication programmes due to lack of availability of rapid serological diagnostic techniques. In most of the conditions, the disease remains subclinical due to long incubation period and may be noticed at the end of life span of sheep during meat inspection or necropsy.

JSRV induces neoplastic transformation of alveolar and bronchiolar secretory epithelial cells. For the first time, York et al. [5] [6] cloned and sequenced the Jaagsiekte retrovirus, the etiological agent of OPA. Later, it was confirmed that JSRV was both necessary and sufficient to induce OPA [7] [8]. In addition to its importance as a veterinary problem, OPA has wider relevance for fundamental studies on cancer since it provides a new model for understanding the molecular events involved in the development of epithelial cell tumors [9]. JSRV infection typically has a serious impact on affected flocks and studies on OPA are an excellent model for human lung carcinogenesis [10]. The main constraint in the diagnosis of OPA is the absence of JSRV-specific antibodies in infected sheep and the lack of availability of serological tests has greatly hindered the development of diagnostic assays [11]-[13]. However, PCR techniques are useful for molecular diagnosis and confirmation of OPA [14]-[17]. Much attention was not given to OPA, though the disease is prevalent in India and the studies were limited to the description of pathomorphological changes. There were no reports on molecular detection and epidemiological aspects of the virus. Hence, the present study was carried out to know the molecular diversity of the JSRV and phylogenetic relationships by detecting JSRV proviral DNA in OPA affected sheep tissues and blood and by partial sequencing of $U 3$ gene of JSRV. This report describes the results of a preliminary study carried out on molecular epidemiological aspects of JSRV in the study area.

\section{Materials and Methods}

\subsection{Study Area and Source of Samples}

The study was conducted from August 2013 to February 2014 in and around Gannavaram town, Andhra Pradesh, India. Samples like lung tumor tissues, mediastinal lymph nodes, blood and lung fluids were collected for gross, histopathological and PCR examinations from OPA suspected sheep from slaughter houses or during necropsy. OPA was diagnosed based on clinical signs, gross and histopathology initially and further confirmed by hn-PCR examination of different samples. For histopathology, tissue samples from lung and mediastinal lymph nodes were processed by paraffin embedding and the sections were stained by $\mathrm{H} \& \mathrm{E}$ staining. Six naturally infected OPA cases were included in the sequencing studies.

\subsection{DNA Extraction}

From lung tumor, mediastinal lymph nodes, blood and lung fluid samples, genomic DNA was extracted by using DNA extraction kit (Qiagen) as per manufacturer's protocol and stored at $-20^{\circ} \mathrm{C}$ until used for PCR.

\subsection{Hemi-Nested (hn) PCR}

U3-hn-PCR was carried out as described previously [15] by using the primers P-I

(TGGGAGCTCTTTGGCAAAAGCC), P-III (CACCGGATTTTTACACAATCACCGG) and P-VI

(TGATATTTCTGTGAAGCAGTGCC) designed for exogenous JSRV [15] and with slight modifications in the PCR programme. Appropriate negative controls were also included in the PCR reactions. In the first round of PCR, 500 ng of genomic DNA was used as template and was added to the PCR master mix (Qiagen) along with primers I and III. The PCR cycles consisted of one cycle of initial denaturation at $94^{\circ} \mathrm{C}$ for 3 min followed by 35 cycles of denaturation at $94^{\circ} \mathrm{C}$ for $30 \mathrm{~s}$, annealing at $61^{\circ} \mathrm{C}$ for $90 \mathrm{~s}$, extension at $72^{\circ} \mathrm{C}$ for 1 min and one cycle of final extension at $72^{\circ} \mathrm{C}$ for $4 \mathrm{~min}$. The PCR amplicon was resolved by electrophoresis in $2 \%$ agarose gel. The DNA band was eluted from gel by using gel extraction kit (Qiagen). This extracted DNA was subjected to second round of PCR by using primers P-I and P-VI and the PCR cycles employed were one cycle of initial denaturation at $94^{\circ} \mathrm{C}$ for $3 \mathrm{~min}$, followed by 35 cycles of denaturation at $94^{\circ} \mathrm{C}$ for $30 \mathrm{~s}$, annealing at $58^{\circ} \mathrm{C}$ for $90 \mathrm{~s}$, extension at $72^{\circ} \mathrm{C}$ for $1 \mathrm{~min}$ and final extension at $72^{\circ} \mathrm{C}$ for $4 \mathrm{~min}$. The PCR amplicon was stained with ethidium 
bromide and resolved in $2 \%$ agarose gel electrophoresis along with 100 bp DNA marker. The PCR products were purified by using gel extraction kit (Qiagen) and the eluted DNA was sequenced by using Sanger Sequencing method by a commercial company (Genotypic Technology Pvt. Ltd., Bangalore, India). The sequences were aligned by using DNASTAR and MEGA 6 software and phylogenetic tree was developed. The sequences were submitted to DNA Data Bank of Japan (DDBJ) and were assigned accession numbers.

\section{Results}

The OPA affected sheep were adult animals, aged about 4 - 5 years and were weak with respiratory distress. On "wheel-barrow" test [18] i.e., when the head was lowered and hind limbs were raised, out flow of mucus discharges from the nostrils were noticed (Figure 1).

Grossly, areas of diffuse consolidation or discrete nodules were noticed in the lungs of infected animals (Figure 2). On sectioning of lung, the surface was moist and greyish white and in one sheep (No. 5), oozing of mucus secretions was more abundant (Figure 3). The mediastinal lymph nodes were slightly enlarged and edematous in appearance. Histologically, the lung sections showed focal or diffuse non encapsulated neoplastic areas with proliferation of alveolar and bronchiolar epithelial cells, either in papillary or acinar pattern (Figure 4).

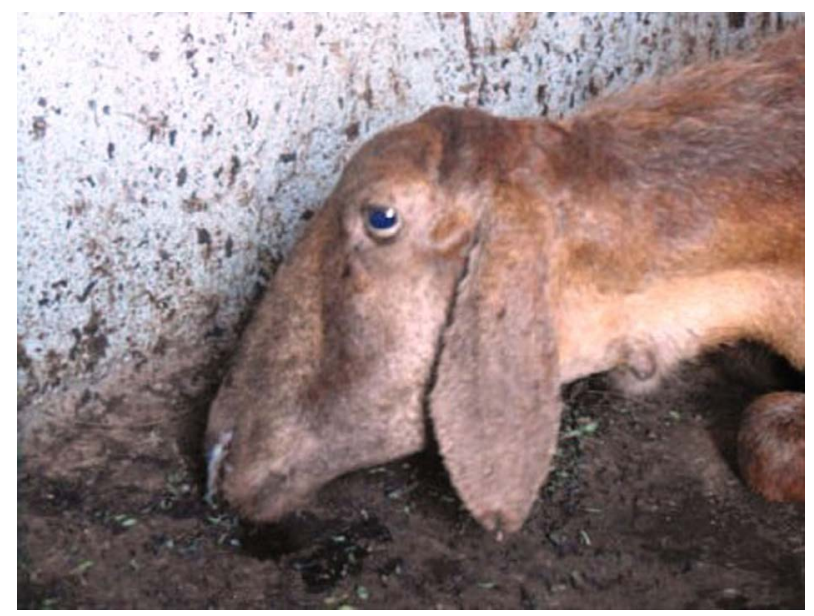

Figure 1. OPA affected sheep with respiratory distress and mucus discharges from the nostrils.

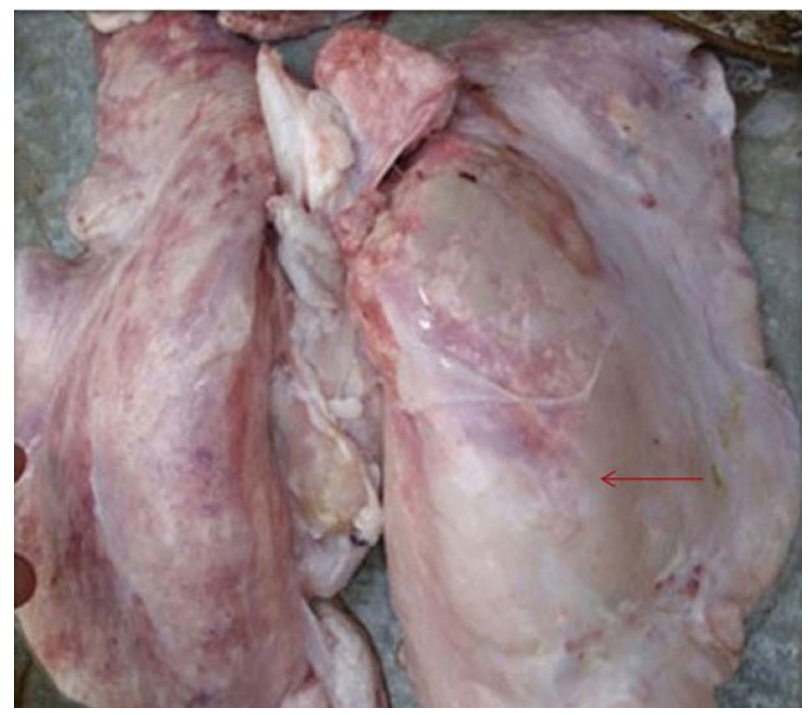

Figure 2. OPA affected lung showing area of diffuse consolidation on diaphragmatic lobe. 


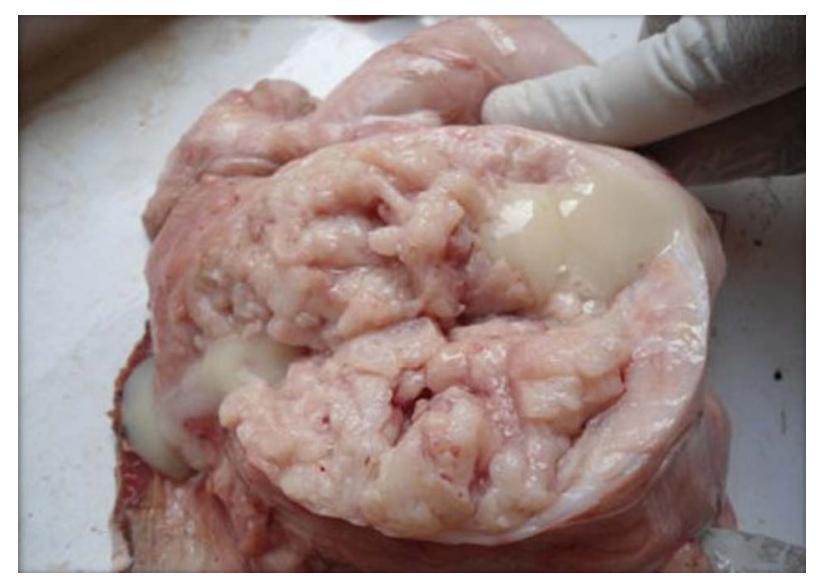

Figure 3. Cut section of consolidated area of OPA affected lung having mucus secretion (sheep No. 5).

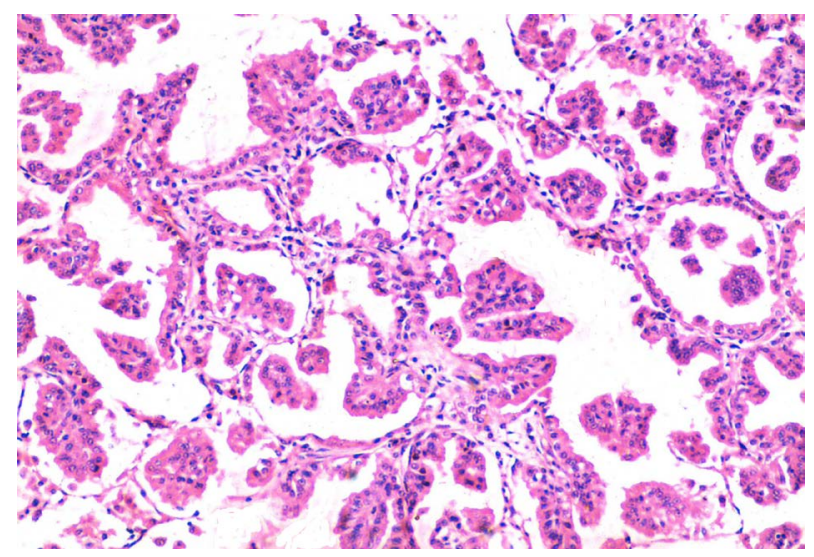

Figure 4. OPA lung showing proliferation of alveolar epithelium with papillary projections. $\mathrm{H} \& \mathrm{E} \times 100$.

The alveoli were lined by cuboidal to columnar epithelial cells. There was hyperplasia of bronchiolar epithelium and polypoid ingrowths of cells were observed into the bronchiolar lumen. Lymphoid cell infiltration in the stroma and around the bronchioles was noticed. In sheep No. 5, the lung sections revealed elongated tubular structures with adenomatous appearance lined by mucin secreting columnar cells (Figure 5). Droplets of mucin was noticed in the cytoplasm of the cells. In sheep No. 6, extensive fibrous tissue proliferation and myxomatous nodules were present in the interstitial tissue (Figure 6). There were no metastatic lesions in the lymph nodes.

U3-hn-PCR amplified 176 bp size of amplicon in first round and $133 \mathrm{bp}$ in the second round of PCR (Figures 7-9). There was no amplification in the negative controls. The eluted DNA from the second round PCR was sequenced and the nucleotide sequences of LTR $U 3$ gene from all six sheep isolates (Figure 10) were submitted to DNA Data Bank of Japan (DDBJ) and were assigned accession numbers (AB914802, AB915878 to AB915882).

The multiple nucleotide sequence alignment of JSRV isolates revealed 96\% - 100\% similarity with a strain from UK (AF105220.1) and 88\% - 93\% homology with a South African strain (M80216). On phylogenetic analysis, the JSRV isolates were segregated into two clusters, with five isolates in one cluster and one isolate in other cluster (Figure 11).

\section{Discussion}

OPA was first reported in India during 1960 [1] and later on it was reported from different parts of the country [2]-[4]. The clinical symptoms, gross and histopathological lesions observed in the OPA affected sheep were similar to the earlier reports [4] [19]-[23]. The affected animals were anorectic and showed polypnea, dyspnea, cough and often pyrexia in the terminal stages of the disease and they were dead within 3 weeks [1]. The 


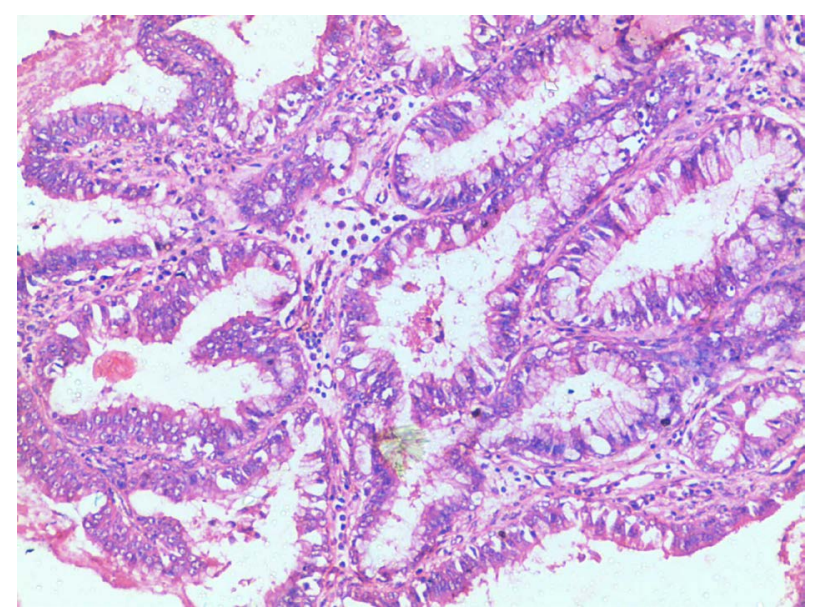

Figure 5. OPA lung (sheep No. 5) revealing tubular structures lined by mucus secreting columnar cells. H \& E ×100.

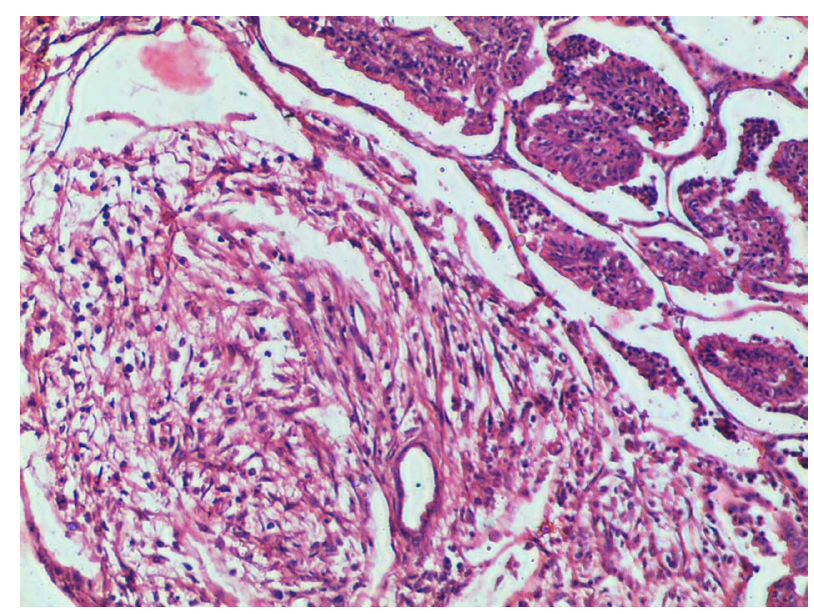

Figure 6. OPA lung (sheep No. 6) showing myxomatous nodule. $\mathrm{H} \& \mathrm{E} \times 100$.

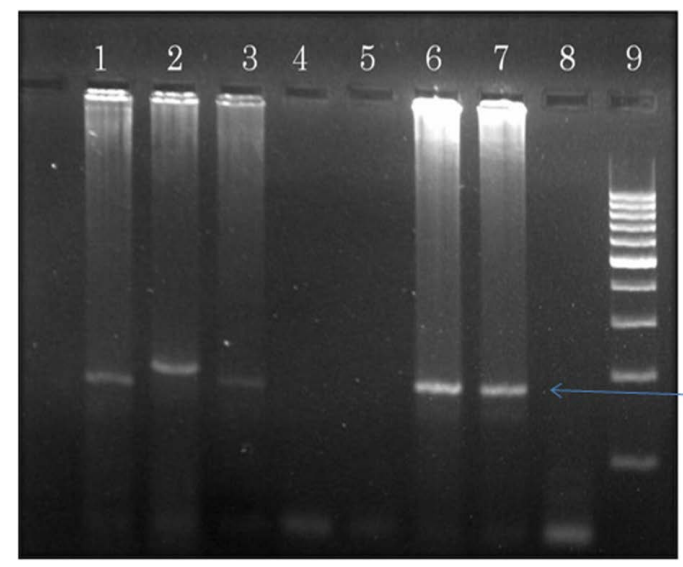

$176 \mathrm{bp}$

Figure 7. Amplification of $176 \mathrm{bp}$ product by first round of hn-PCR for $U 3$ gene in OPA affected animals. Lanes 1, 2, 3, 6, 7 are positive samples; Lanes 4 \& 5-DNA from blood of healthy sheep (Negative); Lane 8-Negative control; Lane 9 -100 bp DNA marker. 


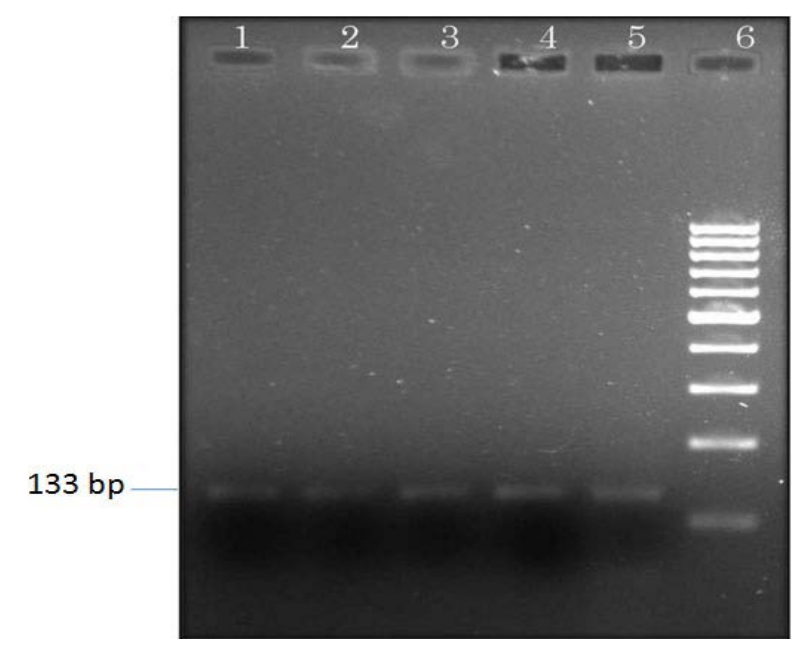

Figure 8. Amplification of 133 bp product by second round of hn-PCR for $U 3$ gene in OPA affected animals. Lanes 1, 2, 3, 4, 5 are positive samples; Lane 6 - 100 bp DNA marker.

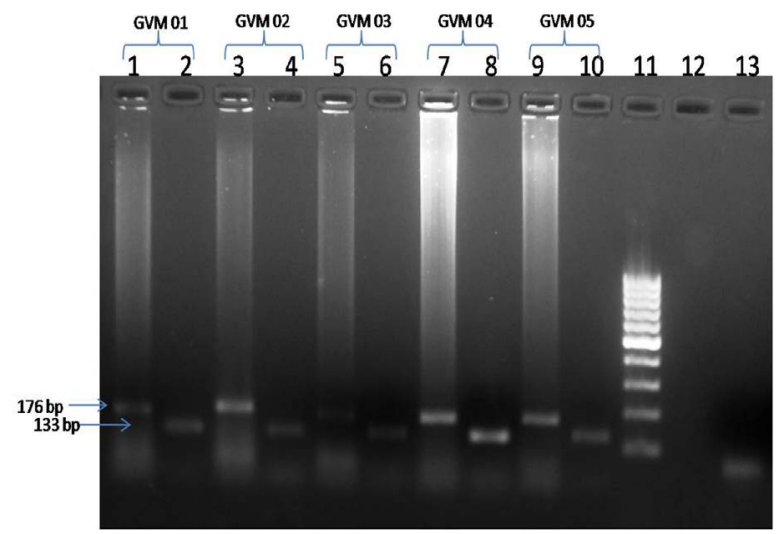

Figure 9. Specific amplification of $U 3$ gene by using hn-PCR in OPA affected animals. Lane 1 \& 2 -Isolate GVM 01; Lane 3 \& 4-Isolate GVM 02; Lane 5 \& 6-Isolate GVM 03; Lane 7 \& 8-Isolate GVM 04; Lane 9 \& 10-Isolate GVM 05; Lane 11-100 bp DNA marker; Lane 12-Primer control; and Lane 13-Negative control.

$>\mathrm{UK}$

STRAIN : TGGGAGCTCTTTGGCAGAAGCCAAAGCCTAGGACAAGTACCTAAGCTCCCTGTCCCGCCACCCTCAAGAATTTTTAAAAGCTCTTAAGGCTCGGATGTTT -GCTTTTGGCACTGCTTCATAGAAATACCA--

>GVM01:TGGGAGCTCTTTGGCAAAAGCCAAAGCCTAGGACAAGTACCTAAGCTCCCTGTCCCGCCACCCTCAAGAATTTTTAAAAGCTCTTAAGGCTTGGAT GTTT-GCTTTTGGCACTGCTTCACAGAAATATCA--

>GVM02:TGGGAGCTCTTTGGCAAAAGCCAAAGCCTAGGACAAGTACCTAAGCTCCCTGTCCCGCCACCCTCAAGAATTTTTAAAAGCTCTTAAGGCTTGGAT GTTT-GCTTTT-- -

>GVM03:TGGGAGCTCTTTGGCAAAAGCCAAAGCCTAGGACAAGTACCTAAGCTCCCTGTCCCGCCACCCTCAAGAATTTTTAAAAGCTCTTAAGGCTTGGAT GTTT-GCTTTTGGCACTGCTTCACAGAAATA-CA--

$>$ GVM0 4 :TGGGAGCTCTTTGGCAAAAGCCAAAGCCTAGGACAAGTACCTAAGCTCCCTGTCCCGCCACCCTCAAGAATTTTTAAAAGCTCTTAAGGCTTGGAT GTTT-GCTTTTGGCACTGCTTCACAGAAATA-----

>GVM0 5:TGGGAGCTCTTTGGCAAAAGCCAAAGCCTAGGACAAGTGCCTAAGCTCCCTGTCCCGCCACCCTCAAGAATTTTTAAAAGCTCCTAAGGCTCGGAT GTTTTGCTTTTGGCACTGCTTCATAGAAATACCA--

>GVM0 6:TGGGAGCTCTTTGGCAAAAGCCAAAGCCTAGGACAAGTACCTAAGCTCCCTGTCCCGCCACCCTCAAGAATTTTTAAAAGCTCTTAAGGCTTGGAT GTTT-GCTTTTGGCACTGCTTCACAGAAATATCA--

$>\mathrm{SA}$

STRAIN : TGGGAGCTCTTTGGCAAAAGCCAAAGCCTAGGACAAATACCTAAGCTCCCTGTCCCGCCACCCTCTAGGATTCTTGAAAGCTCTTAAGGTTCGGATGTTT -GCTGCCGGCATTACTTCACAGAAATGACGGG

Figure 10. Sequence alignment of $U 3$ gene of JSRV isolates, UK strain (AF105220.1) and South African strain (M80216). 


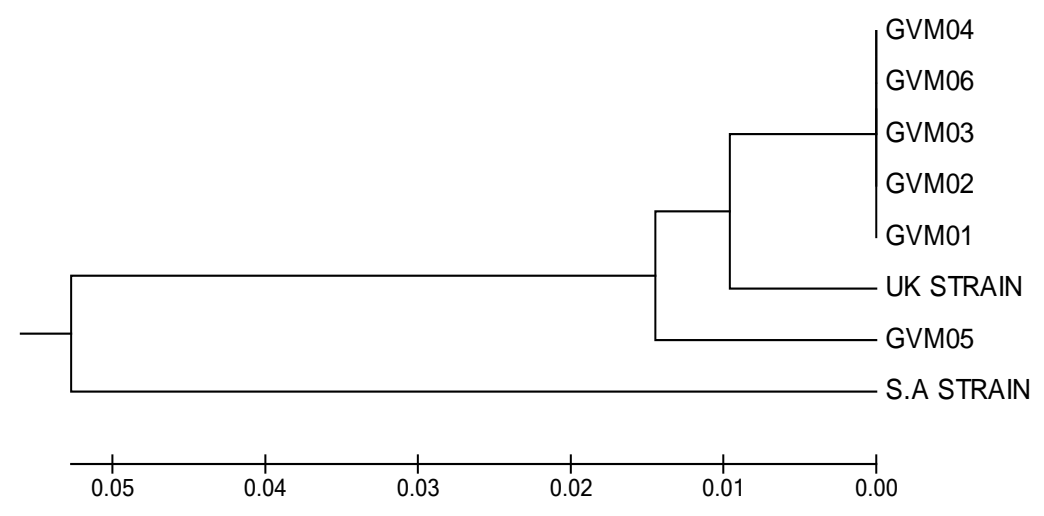

Figure 11. Phylogenetic analysis of JSRV isolates based on $U 3$ gene. Reference strains are UK strain (AF105220.1) and South African strain (M80216).

pathognomonic feature of OPA was the accumulation of lung fluid within the respiratory tract, which may flow from the nostrils when the hind quarters were raised [20]. In the present study, all the OPA sheep showed mucus discharges from the nostrils that was more abundant in sheep No. 5. Grossly, diffuse consolidated areas or discrete nodules seen in the affected lungs with moist surfaces were similar to that of classical form of OPA [1] [19] [23]-[25]. There are descriptions of classical and atypical forms of OPA in the literature [23]-[25]. Three anatomical forms consisting of grey nodules with moist surface in classical form, white, dry and firm nodules clearly demarcated, either solitary or multiple in atypical form and both characteristics in mixed form were described in lungs for the first time by De Las Heras et al. [24] Later, the classical form of OPA characterized by a firm, greyish to purple neoplastic mass often surrounded by satellite tumor nodules with the cut section of tumor mass invariably having a wet appearance and copious fluid filling the airways and the atypical form showing a solitary tumor nodule in the diaphragmatic lobe and several smaller nodules in the main lobes, with hard and pearly white and a dry cut surface were reported [25]. On histological examination, the lung sections from consolidated areas or tumor nodules revealed proliferation of alveolar or bronchiolar epithelial cells giving either papillary or acinar pattern. These lesions were in accordance with the observations of previous reports [1] [4] [22] [23] [26]. Garcia-Goti et al. [25] observed similar histological changes in both classical and atypical forms of OPA in lungs. In natural or experimentally induced OPA, the neoplastic cells formed acinar structures or papillary proliferations [26] as observed in the present study and the myxomatous nodules noticed in the OPA lung in sheep No. 6 were also reported previously [19] [25].

In the present study, U3-hn-PCR was conducted to confirm the presence of JSRV proviral DNA in the infected sheep samples. The primers developed from JSRV LTR U3 were used in hn-PCR to amplify JSRV proviral sequences as described previously [15] as there are sequence differences in U3 region of LTR of exJSRV that can differentiate both exJSRV and endogenous JSRV (enJSRV). The unique U3 sequences are the hallmark for all exJSRV proviruses that are detectable in ovine pulmonary carcinoma lung tumor DNA [27]. The most remarkable sequence difference between the LTR sequences was that the endogenous U3 region was 47 bp longer than that of exJSRV [6]. There are twenty seven enJSRV related proviruses in sheep genome [28] [29] and they have over $90 \%$ sequence similarity with exJSRV in most parts of the genome [15] [27]-[30]. However, enJSRVs cannot produce tumors as most of them are defective and silent, differing from exJSRV. Because of sequence divergence in U3 region, the primers developed from that area can differentiate exJSRV and enJSRV proviruses and can be used for diagnostic confirmation of OPA. The results of U3-hn-PCR and sequencing of PCR amplicons in the present study confirmed the presence of $U 3$ gene of exJSRV in the infected sheep samples. The nucleotide sequence analysis of six JSRV isolates from the OPA infected sheep showed more similarity with a UK strain (AF105220.1) that was cloned from a lung tumor tissue by Palmarini et al. [7] than with a South African strain (M80216), the JSRV strain which was cloned and sequenced for the first time [6]. The phylogenetic analysis revealed divergence among the isolates and the sheep isolate GVM 05 was more divergent when compared with others. The gross and histological lesions of lung in sheep No. 5, have also showed some variation with lot of mucus secretion and presence of predominantly tubular structures lined by mucus secreting columnar cells, when compared to papillary type of lesions in other animals. However, studies like cloning and 
sequencing of full genome of JSRV in Indian sheep are required for further understanding in this aspect. The nucleotide sequencing and phylogenetic analysis of six Indian isolates of JSRV using $U 3$ gene is the first report and these findings will be useful for future studies.

\section{Conclusion}

In conclusion, this study is the first report on nucleotide sequencing and phylogenetic analysis of JSRV in India and it was revealed that the isolates from Indian sheep had homology with a UK strain and divergent from a South African strain and were segregated into two clusters.

\section{Acknowledgements}

The financial assistance given by SERB, Department of Science and Technology (DST), Govt. of India and the facilities to carry out the work provided by Sri Venkateswara Veterinary University, Tirupati are acknowledged.

\section{References}

[1] Damodaran, S. (1960) Ovine Pulmonary Adenomatosis (Jagziekte). Indian Veterinary Journal, 37, 127-138.

[2] Razya, B.S. and Singh, C.M. (1964) The Pathology of Pneumonia and Associated Respiratory Disease of Sheep and Goats. I. Occurrence of Jagziekte and Maedi in Sheep and Goats in India. American Journal of Veterinary Research, 25, 61-67.

[3] Sarkar, C.R., Chakrabarti, A., Deb, S. and Nandy, S.N. (1988) Pulmonary Adenomatosis (Jaagsiekte) of Sheep in West Bengal. Indian Veterinary Journal, 65, 353-354.

[4] Rama Devi, V., Srilatha, Ch., Sujatha, K. and Nisar Ahmed, K.N. (2001) Pulmonary Adenomatosis in Sheep-A Case Report. Indian Veterinary Journal, 78, 853-854.

[5] York, D.F., Vigne, R., Verwoerd, D.W. and Querat, G. (1991) Isolation, Identification, and Partial cDNA Cloning of Genomic RNA of Jaagsiekte Retrovirus, the Etiological Agent of Sheep Pulmonary Adenomatosis. Journal of Virology, 65, 5061-5067.

[6] York, D.F., Vigne, R., Verwoerd, D.W. and Querat, G. (1992) Nucleotide Sequence of the Jaagsiekte Retrovirus, an Exogenous and Endogenous Type D and B Retrovirus of Sheep and Goats. Journal of Virology, 66, 4930-4939.

[7] Palmarini, M., Sharp, J.M., De Las Heras, M. and Fan, H. (1999) Jaagsiekte Sheep Retrovirus Is Necessary and Sufficient to Induce a Contagious lung Cancer in Sheep. Journal of Virology, 73, 6964-6972.

[8] De Martini, J.C., Bishop, J.V., Allen, T.E., Jassim, F.A., Sharp, J.M., De Las Heras, M., Voelkr, D.R. and Carlson, J.O. (2001) Jaagsiekte Sheep Retrovirus Proviral Clone JSRV ${ }_{\mathrm{js}}$, Derived from the JS7 Lung Tumor Cell Line, Induces Ovine Pulmonary Carcinoma and Is Integrated into the Surfactant Protein A Gene. Journal of Virology, 75, 4239-4246. http://dx.doi.org/10.1128/JVI.75.9.4239-4246.2001

[9] Palmarini, M. and Fan, H. (2001) Retrovirus-Induced Ovine Pulmonary Adenocarcinoma, an Animal Model for Lung Cancer. Journal of the National Cancer Institute, 93, 1603-1614. http://dx.doi.org/10.1093/jnci/93.21.1603

[10] Hofacre, A. and Fan, H. (2010) Jaagsiekte Sheep Retrovirus Biology and Oncogenesis. Viruses, 2, 2618-2648. http://dx.doi.org/10.3390/v2122618

[11] Sharp, J.M., Angus, K.W., Gray, E.W. and Scott, F.M. (1983) Rapid Transmission of Sheep Pulmonary Adenomatosis (Jaagsiekte) in Young Lambs. Archives of Virology, 78, 89-95. http://dx.doi.org/10.1007/BF01310861

[12] Ortín, A., Minguijón, E., Dewar, P., García, M., Ferrer, L.M., Palmarini, M., Gonzalez, L., Sharp, J.M. and De Las Heras, M. (1998) Lack of a Specific Immune Response against a Recombinant Capsid Protein of Jaagsiekte Sheep Retrovirus in Sheep and Goats Naturally Affected by Enzootic Nasal Tumour or Sheep Pulmonary Adenomatosis. Veterinary Immunology and Immunopathology, 61, 229-237. http://dx.doi.org/10.1016/S0165-2427(97)00149-9

[13] Summers, C., Neill, W., Dewar, P., Gonzalez, L., van der Molen, R., Norval, M. and Sharp, J.M. (2002) Systemic Immune Responses Following Infection with Jaagsiekte Sheep Retrovirus and in the Terminal Stages of Ovine Pulmonary Adenocarcinoma. Journal of General Virology, 83, 1753-1757.

[14] Gonzalez, L., Garcia-Goti, M., Cousens, C., Dewar, P., Cartobarria Extramiana, N., Ortin, A., De Las Heras, M. and Sharp, J.M. (2001) Jaagsiekte Sheep Retrovirus Can Be Detected in the Peripheral Blood during the Pre Clinical Period of Sheep Pulmonary Adenomatosis. Journal of General Virology, 82, 1355-1358.

[15] Palmarini, M., Holland, M.J., Cousens, C., Dalziel, R.G. and Sharp, J.M. (1996) Jaagsiekte Retrovirus Establishes a Disseminated Infection of the Lymphoid Tissues of Sheep Affected by Pulmonary Adenomatosis. Journal of General Virology, 77, 2991-2998. http://dx.doi.org/10.1099/0022-1317-77-12-2991 
[16] De Las Heras, M., Ortin, A., Salvatori, D., Perez de Villareal, M., Cousens, C., Miguel Ferrer, L., Miguel Cebrian, L., Antonio Garcia de Jalon, J., Gonzalez, L. and Sharp, J.M. (2005) A PCR Technique for the Detection of Jaagsiekte Sheep Retrovirus in the Blood Suitable for Screening of Ovine Pulmonary Adenocarcinoma in Field Conditions. Research in Veterinary Science, 79, 259-264. http://dx.doi.org/10.1016/j.rvsc.2005.02.003

[17] Voigt, K., Brugmann, M., Huber, K., Dewar, P., Cousens, C., Hall, M., Sharp, J.M. and Ganter, M. (2007) PCR Examination of Bronchoalveolar Lavage Sample Is a Useful Tool in Pre-Clinical Diagnosis of Ovine Pulmonary Adenocarcinoma (Jaagsiekte). Research in Veterinary Science, 83, 419-427. http://dx.doi.org/10.1016/j.rvsc.2007.02.001

[18] Sharp, J.M. and De Las Heras, M. (2000) Contagious Respiratory Tumors. In: Martin, W.B. and Aitken, I.D., Eds., Diseases of Sheep, Blackwell Science, Oxford, 181-186.

[19] Rahman, T. and Iyer, P.K.R. (1979) Studies on Pathology of Ovine Pneumonias. Indian Veterinary Journal, 56, 455461.

[20] Salvatori, D., De Las Heras, M. and Sharp, M. (2004) Ovine Pulmonary Adenocarcinoma: The Story to Date. In Practice, 26, 387-392. http://dx.doi.org/10.1136/inpract.26.7.387

[21] Kaycko, A., Jasik, A. and Reichert, M. (2008) Detection of Jaagsiekte Sheep Retrovirus in Respiratory Tract Fluid and Lung Tissue of Experimentally Infected Lambs. Bulletin of the Veterinary Institute in Pulawy, 52, 9-13.

[22] Beytut, E., Sozmen, M. and Erginsoy, S. (2009) Immunohistochemical Detection of Pulmonary Surfactant Proteins and Retroviral Antigens in the Lungs of Sheep with Pulmonary Adenomatosis. Journal of Comparative Pathology, 140, 43-53. http://dx.doi.org/10.1016/j.jcpa.2008.10.003

[23] Khodakaram-Tafti, A. and Razavi, Z. (2010) Morphopathological Study of Naturally Occurring Ovine Pulmonary Adenocarcinoma in Sheep in Fars Province, Iran. Iranian Journal of Veterinary Research, 11, 134-138.

[24] De las Heras, M., Calafat, J.J., Jaime, J.M., Garcia de Jalon, J.A., Ferrer, L.M., Garcia-Goti, M. and Minguijon, E. (1992) Sheep Pulmonary Adenomatosis (jaagsiekte) in Slaughtered Sheep Variation in Pathological Characteristics. Medicina Veterinaria, 9, 52-53.

[25] Garcia-Goti, M., Gonzalez, L., Cousens, C., Cartobarria, N., Extramiana, A.B., Minguijon, E., Ortini, A., De las Heras, M. and Sharp, J.M. (2000) Sheep Pulmonary Adenomatosis Characterization of Two Pathological Forms Associated with Jaagsiekte Retrovirus. Journal of Comparative Pathology, 122, 55-65. http://dx.doi.org/10.1053/jcpa.1999.0344

[26] Platt, J.A., Kraipowich, N., Villafane, F. and DeMartini, J.C. (2002) Alveolar Type II Cells Expressing Jaagsiekte sheep Retrovirus Capsid Protein and Surfactant Proteins Are the Predominant Neoplastic Cell Types in Ovine Pulmonary Adenocarcinoma. Veterinary Pathology, 39, 341-352. http://dx.doi.org/10.1354/vp.39-3-341

[27] Bai, J., Zhu, R.Y., Stedman, K., Cousens, C., Carlson, J., Sharp, J. and DeMartini, J.C. (1996) Unique Long Terminal Repeat U3 Sequences Distinguish Exogenous Jaagsiekte Sheep Retroviruses Associated with Ovine Pulmonary Carcinoma from Endogenous Loci in the Sheep Genome. Journal of Virology, 70, 3159-3168.

[28] Bai, J., Bishop, J.V., Carlson, J. and DeMartini, J.C. (1999) Sequence Comparison of JSRV with Endogenous Proviruses: Envelope Genotypes and a Novel ORF with Similarity to a G-Protein-Coupled Receptor. Virology, 258, $333-343$. http://dx.doi.org/10.1006/viro.1999.9728

[29] Palmarini, M., Datta, S., Omid, R., Murgia, C. and Fan, H. (2000) The Long Terminal Repeat of Jaagsiekte Sheep Retrovirus Is Preferentially Active in Differentiated Epithelial Cells of the Lungs. Journal of Virology, 74, 5776-5787. http://dx.doi.org/10.1128/JVI.74.13.5776-5787.2000

[30] Arnaud, F., Murcia, P.R. and Palmarini, M. (2007) Mechanisms of Late Restriction Induced by an Endogenous Retrovirus. Journal of Virology, 81, 11441-11451. http://dx.doi.org/10.1128/JVI.01214-07 
Scientific Research Publishing (SCIRP) is one of the largest Open Access journal publishers. It is currently publishing more than 200 open access, online, peer-reviewed journals covering a wide range of academic disciplines. SCIRP serves the worldwide academic communities and contributes to the progress and application of science with its publication.

Other selected journals from SCIRP are listed as below. Submit your manuscript to us via either submit@scirp.org or Online Submission Portal.
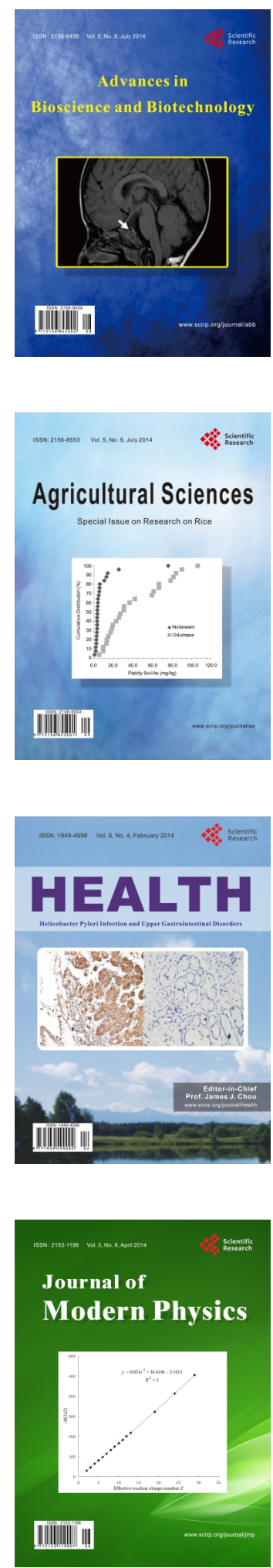
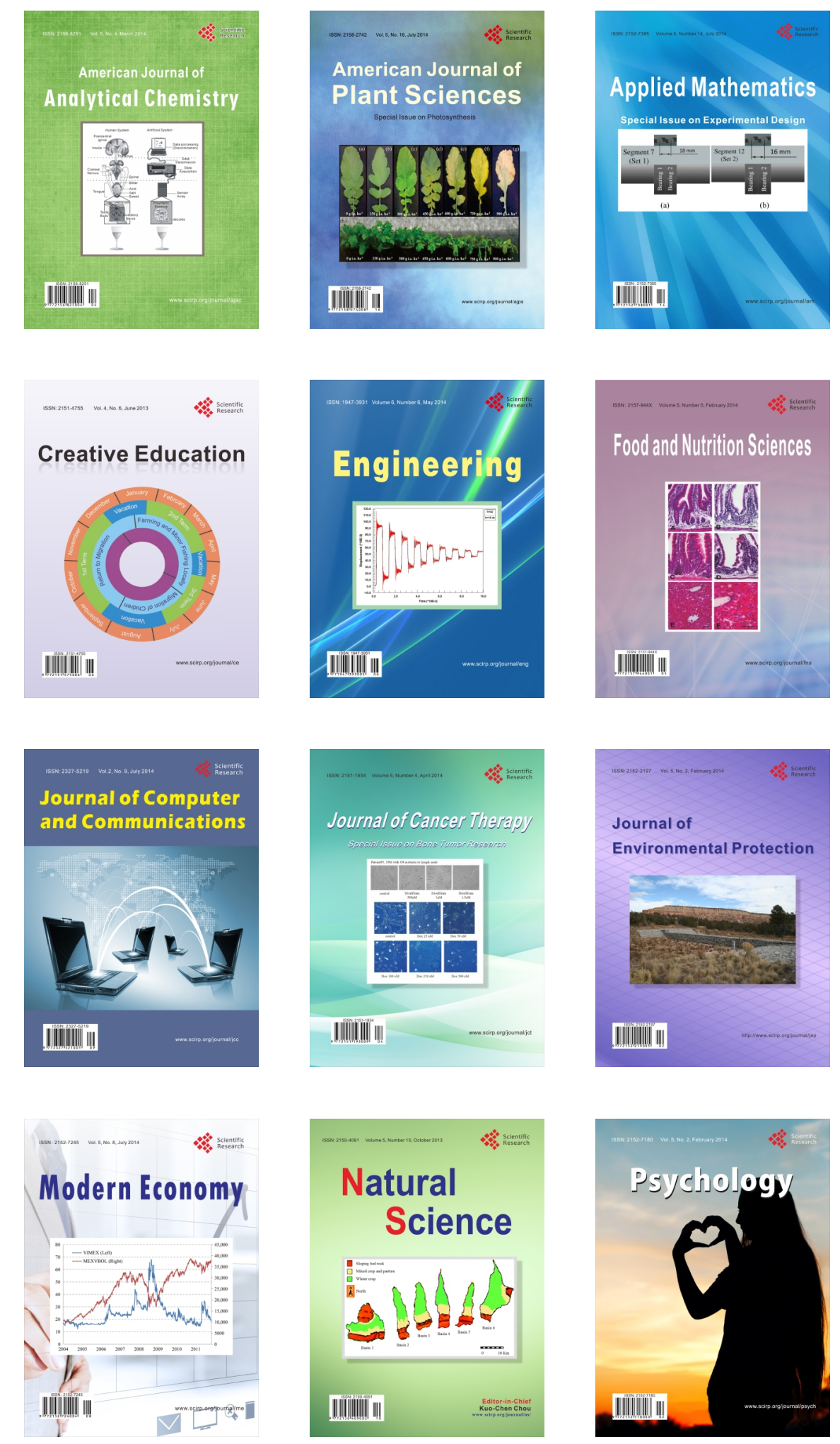